\title{
La evolución de paradigmas en las investigaciones de ocurrencias aeronáuticas
}

\author{
Evolution of paradigms in aeronautical occurrences' investigations
}

A evolução de paradigmas nas investigações de ocorrências aeronáuticas

\author{
Ten Cel Av Adalberto Santos Prado \\ Cuarto Servicio Regional de Investigación y Prevención \\ de Accidentes Aeronáuticos - SERIPA IV \\ São Paulo/SP - Brasil \\ adalbertosprado@gmail.com
}

Cel Av R1 Flavio Neri Hadmann Jasper, Doctor Secretaria de Economía y Finanzas de Aeronáutica - SEFA

Brasília/DF - Brasil fnhjasper@gmail.com

\section{RESUMEN}

Con el avance de las ciencias aeronáuticas, se tornaron necesarias nuevas teorías sobre investigación de accidentes aéreos para explicar y promover la prevención de esas ocurrencias. Las teorías que buscan explicar los procesos involucrados en los accidentes aeronáuticos sufrieron evoluciones, de acuerdo con las diferentes realidades y desarrollos de la aviación en las diversas épocas, sin embargo no hubo una ruptura total. Heinrich (1931) publicó la teoría de la causa única, con la existencia de una acción insegura, cercana al accidente, que debería ser impedida para que no hubiese un daño. Ese pensamiento fue representado por el modelo del dominó y fue muy importante en el surgimiento de las teorías siguientes. Reason (1997) desarrolló la teoría de las causas múltiples, representada por el modelo del Queso Suizo. Según esa teoría, en las organizaciones, hay condiciones latentes que actúan en las vulnerabilidades de las defensas y, cuando son alineadas y asociadas a una falla activa, provocan el accidente. Como resultado de la evolución de paradigmas en las investigaciones de ocurrencias aeronáuticas, actualmente, los aspectos organizativos son considerados en los procesos de investigación y en los programas de prevención de accidentes aeronáuticos, así como en la identificación de peligros o amenazas. Este concepto fundamenta la administración del riesgo, manteniendo dentro de un nivel aceptable y compatible con el desarrollo de la actividad aérea. Esas evoluciones de paradigmas en los procesos de seguridad de vuelo fueron analizadas a la luz de la teoría de las revoluciones científicas de Kuhn (1991), a través de investigación descriptiva de dos tipos: la bibliográfica, consultando fuentes teóricas, como artículos científicos, libros, diccionarios y periódicos, y la documental.

Palabras clave: Investigación. Seguridad. Paradigma. Accidente.

Recibido / Received / Recebido $19 / 08 / 14$
Aceptado / Accepted / Aceito $11 / 04 / 15$ 


\begin{abstract}
With the advance of aeronautical sciences, new theories on air accident investigation were necessary to explain and promote the prevention of these occurrences. Theories that attempt to explain processes involved in aeronautical accidents have suffered evolutions, according to the different realities and aviation development in different times; however, there was no total rupture. Heinrich (1931) published the single cause theory, stating the existence of one unsafe action, next to the accident, which should have been hindered to avoid the damage occurrence. This thought was represented by the domino model and was very important to the appearance of subsequent theories. Reason (1997) developed the theory of multiple causes, represented by the Swiss cheese model. According to that theory, in organizations, there are latent conditions which act on the defense of vulnerabilities and, when aligned with and associated to an active failure, cause the accident. As a result of the evolution of paradigms in aeronautical occurrences' investigations, today, organizational aspects are considered in investigation process and programs for prevention of aeronautical accidents, as well as the identification of hazards or threatens. This concept grounds risk management, keeping it within an acceptable level compatible with the development of air activities. Those evolutions of paradigms in flight safety processes were analyzed in the light of the Kuhn's (1991) scientific revolutions theory, by means of descriptive research of two types: the bibliographic, with consultation to theoretical sources like scientific papers, books, dictionaries and periodicals, and the documentary.
\end{abstract}

\title{
Keywords: Investigation. Safety. Paradigm. Accident. RESUMO
}

Com o avanço das ciências aeronáuticas, novas teorias sobre investigação de acidentes aéreos tornaram-se necessárias para explicar e promover a prevenção destas ocorrências. As teorias que procuram explicar os processos envolvidos nos acidentes aeronáuticos sofreram evoluções, de acordo com as diferentes realidades e desenvolvimento da aviação nas diversas épocas, porém não houve uma ruptura total. Heinrich (1931) publicou a teoria da causa única, com a existência de uma ação insegura, próxima ao acidente, que deveria ser impedida para que não ocorresse o dano. Esse pensamento foi representado pelo modelo do dominó e foi muito importante no surgimento das teorias seguintes. Reason (1997) desenvolveu a teoria das causas múltiplas, representada pelo modelo do queijo suíço. Segundo essa teoria, nas organizações, há condições latentes que atuam nas vulnerabilidades das defesas e, quando alinhadas e associadas a uma falha ativa, provocam o acidente. Como resultado da evolução de paradigmas nas investigações de ocorrências aeronáuticas, atualmente, os aspectos organizacionais são considerados nos processos de investigação e nos programas de prevenção de acidentes aeronáuticos, assim como na identificação de perigos ou ameaças. Esse conceito fundamenta o gerenciamento do risco, mantendo-o dentro de um nível aceitável e compatível com o desenvolvimento da atividade aérea. Essas evoluções de paradigmas nos processos de segurança de voo foram analisadas à luz da teoria das revoluções científicas de Kuhn (1991), através de pesquisa descritiva de dois tipos: a bibliográfica, consultando-se fontes teóricas, como artigos científicos, livros, dicionários e periódicos, e a documental.

Palavras-chave: Investigação. Segurança. Paradigma. Acidente.

\section{INTRODUCCIÓN}

El primer accidente aeronáutico que resultó en la primera investigación de accidente aeronáutico de la historia ocurrió el 17 de setiembre de 1908, en la ciudad de Fort Myer, Virgínia. La aeronave accidentada era piloteada por Orville Wright, uno de los hermanos Wright, y el pasajero, Teniente Thomas Selfridge, fue la primera víctima fatal de la aviación mundial (EBER, 1982).

A partir de ese evento trágico, el hombre buscó desarrollar la actividad aérea, a fin de evitar las pérdidas materiales y humanas. Bajo la perspectiva de prevención de esas pérdidas, diversas teorías intentaron explicar la ocurrencia de accidentes aeronáuticos. A partir de nuevos abordajes, otros métodos preventivos fueron desarrollados, ya que la investigación de ocurrencias es una herramienta reactiva importante para la seguridad de vuelo.
De esa forma, este trabajo tiene como objetivo analizar los cambios en los procesos de investigación de accidentes aeronáuticos, considerando el abordaje teórico propuesto por Kuhn (1991) para explicar las estructuras de las revoluciones científicas.

\section{INVESTIGACIÓN DE ACCIDENTES AERONÁUTICOS EN BRASIL}

Inicialmente, en el desarrollo de las actividades aeronáuticas en Brasil predominaba la aviación militar. Consecuentemente, las investigaciones de las ocurrencias, militares o no, eran hechas por el Ejército Brasileño, por medio de Investigación Policial Militar (IPM), y por la Marina de Brasil, por medio de Investigación de Accidente Aeronáutico (IAA). La principal finalidad de esos cálculos era la responsabilidad de lo ocurrido. 
En 1941, fue creado el Ministerio de Aeronáutica siendo la Inspección General de Aeronáutica responsable por las investigaciones de accidentes aeronáuticos. La Investigación de Accidente Aeronáutico y la Investigación Policial Militar fueron sustituidas por la Investigación Técnico Sumaria (ITS), con el objetivo de proporcionar mayor agilidad a las investigaciones de accidentes aeronáuticos.

El Servicio de Investigación de Accidentes Aeronáuticos fue establecido el 5 de abril de 1948, por medio del Decreto $\mathrm{n}^{\circ} 24.749$, definiendo el objetivo de las investigaciones. En ese documento, fue prevista la adopción de medidas preventivas o represivas para evitar nuevos accidentes. De esa forma, competía al Comandante de Zona Aérea y a los Comandantes de Unidades imponer sanciones disciplinarias a los responsables por los accidentes, reforzando el carácter punitivo de la seguridad de vuelo en esa época. En el caso de indicio de crimen o contravención, debería ser instaurado IPM paralelamente o después de la investigación aeronáutica, descrita en el Decreto. Fue organizada, en la Tercera División de la Inspección del Estado Mayor de la Aeronáutica, una sección responsable por los asuntos afectos a accidentes aeronáuticos, con la incumbencia de fiscalizar el cumplimiento del Decreto, orientar los Encargados de Accidentes de las unidades y las Comisiones de Accidentes sobre los métodos más adecuados a las investigaciones, sugerir medidas preventivas, recibir y analizar los procesos de accidentes, además de realizar cálculos estadísticos y divulgar los resultados.

El 11 de octubre de 1965, por medio del Decreto $\mathrm{n}^{\circ} 57.055$, hubo un cambio significativo en el modelo de las investigaciones de accidentes aeronáuticos en Brasil, pues los accidentes aeronáuticos pasaron a ser analizados, considerando los factores humanos, material y operativo. El ITS y el Informe Sumario fueron sustituidos por el Informe de Investigación de Accidente Aeronáutico (RELIAA) y por el Informe Final (RELFIN).

Por medio del Decreto $\mathrm{n}^{\circ}$ 69.565, del 19 de noviembre de 1971, la sigla SIPAER pasó a significar Sistema de Investigación y Prevención de Accidentes Aeronáuticos, creando el Centro de Investigación y Prevención de Accidentes Aeronáuticos (CENIPA) como órgano central de ese sistema. A partir de entonces, el término indagación no sería más utilizado en las investigaciones de ocurrencias aeronáuticas y el propósito de las investigaciones se tornó exclusivamente preventivo, según normas internacionales.

En 1973, para separar los procedimientos de investigación de accidentes aeronáuticos con finalidad de prevención de ocurrencias similares de los procedimientos de investigación con finalidad de cálculo de responsabilidades, Brasil sugirió a la International Civil Aviation Organization (ICAO) la sustitución del término indagación por investigación. En 1974, la ICAO aceptó y adoptó la propuesta brasileña.

Actualmente, la NSCA 3-6 (aeronaves militares) y la NSCA 3-13 (aeronaves civiles) establecen los protocolos de investigación de ocurrencias aeronáuticas bajo responsabilidad de Brasil. En el caso de la aviación civil, el CENIPA envía a la ICAO el Final Report y el Accident/Incident Data Reporting (ADREP), cuando la ocurrencia contempla los criterios de gravedad y peso máximo de despegue establecidos internacionalmente. Esas informaciones ayudan a componer la base de datos de la ICAO utilizada para administrar la seguridad de vuelo en el mundo.

La Ley $\mathrm{N}^{\circ}$ 12.970, del 8 de mayo de 2014, dispone sobre las investigaciones realizadas por el SIPAER y establece que el único propósito de esas investigaciones es la prevención de otros accidentes e incidentes. Define, también, que las Recomendaciones de Seguridad pueden ser emitidas en cualquier etapa de la investigación y que serán consideradas inclusive las hipótesis para identificar de factores contribuyentes. Ese aspecto diferencia la investigación SIPAER, con finalidad preventiva, de la investigación policial, que posee otras finalidades. Las hipótesis y demás factores investigados por el SIPAER no obedecen necesariamente a los criterios exigidos para la atribución de responsabilidad civil o criminal, pues defienden la vida, derecho prioritario en el ordenamiento jurídico de Brasil. El sigilo profesional y la protección de la información, previstos en la referida ley, contribuyen para la eficacia de la investigación realizada por el SIPAER, ya que incentivan el suministro voluntario de informaciones importantes para la prevención de nuevos accidentes.

\section{EVOLUCIÓN DE PARADIGMAS EN LA SEGURIDAD DE VUELO MUNDIAL}

\author{
Según Kuhn,
}

paradigmas son las realizaciones científicas universalmente reconocidas que, durante algún tiempo, suministran problemas y soluciones modelo para una comunidad de practicantes de una ciencia. (KUHN, 1991, p.13, nuestra traduccíon).

Bajo esa perspectiva, la evolución de paradigmas en relación a los fundamentos de la seguridad de 
vuelo no ocurrió solamente en relación a la finalidad de las investigaciones de ocurrencias aeronáuticas en Brasil. La aviación, también en otros países, adoptaba una postura fly-crash-fly (vuela-rompe-vuela), o sea, los vuelos eran realizados, hasta que ocurriese algún accidente, hasta que fuese recuperada la aeronave, fuese investigada la ocurrencia, fuesen adoptadas acciones correctivas y se retomase la actividad aérea. Las causas de los accidentes eran atribuidas a las condiciones meteorológicas, a las fallas mecánicas y, generalmente, al error humano, principalmente del piloto. En este último caso, la antigua filosofía recomendaba, básicamente, alentar a los demás pilotos a no cometer el mismo error, mientras el ciclo de prevención iba siendo considerado concluido (STOLZER; HALFORD; GOGLIA, 2008).

Actualmente, la seguridad de vuelo es estudiada sistémicamente. Fueron incorporados conceptos de Safety Management Systems (SMS) - ya utilizados en otros sectores, como la industria química, alimenticia, eléctrica - a los documentos de la ICAO. Esa herramienta fue recomendada a todos los países firmantes de la Convención de Chicago por medio del documento (DOC) $n^{\circ}$ 9859, Safety Management Manual, $1^{a}$ edición, 2006.

Según la ICAO (2013), la historia de la seguridad de vuelo puede ser dividida en tres eras:

Era técnica: hasta el final de la década de 1960, la aviación surgía como forma de transporte y los temas de seguridad eran relacionados a problemas técnicos y tecnológicos. A partir de la década de 1950, el progreso tecnológico trajo una reducción en las estadísticas de accidentes y la seguridad de vuelo incorporó asuntos de cumplimiento de reglas y supervisión.

Era de los factores humanos: desde inicios de la década de 1970 hasta mediados de la década de 1990, la frecuencia de los accidentes aeronáuticos ya había sido reducida, debido a los avances tecnológicos, y la aviación se torna el medio de transporte más seguro. De esa forma, se buscó un nuevo foco para continuar reduciendo las estadísticas de accidentes aeronáuticos. Surgieron estudios sobre la interacción hombre máquina y el desempeño humano en las tareas relacionadas a las actividades aeronáuticas. No había, sin embargo, la comprensión de que el comportamiento humano podría ser influenciado por diversas condiciones ambientales y organizativas, modificando su performance.

Era organizacional: a partir de la segunda mitad de la década de 1990, la seguridad de vuelo pasó a ser vista de forma sistémica, considerando no solamente factores humanos y tecnológicos, sino también organizativos. A partir de entonces, fueron tomados en consideración los impactos de las culturas organizacionales y de las políticas de seguridad en el control de los riesgos, manteniéndolos dentro de un nivel aceptable. La metodología de recolección y análisis de datos también fueron reformuladas. Anteriormente, los estudios de seguridad de vuelo eran basados en informaciones recolectadas después de eventos negativos, básicamente accidentes e incidentes graves, medidas esencialmente reactivas. Por medio de una perspectiva proactiva, se pasó a monitorear constantemente los indicadores de niveles de seguridad, identificar los peligros o amenazas y administrar los riesgos, buscando prevenir accidentes.

Las evoluciones de paradigmas en la seguridad de vuelo pueden ser analizadas bajo la óptica de la teoría de Kuhn (1991, p.22): “el desarrollo de la mayoría de las ciencias se ha caracterizado por la continua competencia entre diversas concepciones de naturaleza distintas", según descrito por la ICAO (2013), o sea:

Inicialmente, los accidentes aeronáuticos eran investigados considerando factores tecnológicos. A partir de determinado momento, el abordaje tecnológico no era más suficiente para reducir la estadística de accidentes aeronáuticos y los factores humanos pasaron a constituir un nuevo paradigma en la prevención de accidentes aeronáuticos. Posteriormente, se constató que la investigación dirigida al hombre, sin considerar los factores organizacionales y operativos que influencian su comportamiento, no bastaría para explicar los accidentes aeronáuticos y promover la seguridad de vuelo, lo que originó nuevo abordaje del tema.

Se destaca que en las transiciones entre las eras históricas de la aviación no hubo una ruptura, sino una evolución de paradigmas, ya que las nuevas perspectivas no serían posibles sin las experiencias anteriores. Por ejemplo, aunque actualmente la seguridad de vuelo procure actuar proactivamente, no fueron abandonadas las prácticas reactivas de investigación de accidentes aeronáuticos.

Por más que sean considerados factores organizacionales en las medidas preventivas, aspectos tecnológicos y factores humanos no fueron descartados. En relación a las teorías sobre la ocurrencia de los accidentes aeronáuticos, también hubo evolución de paradigmas en el modelo.

Heinrich (1931) creó la teoría de la causa única o teoría del dominó. Se trata de un modelo lineal de tipo causaefecto, en el cual la investigación estaría focalizada en los factores más íntimamente relacionados a los accidentes, conforme representado en la Figura 1. Heinrich (1931) no consideraba provechoso, por ejemplo, investigar los más altos niveles administrativos. Defendía que sería posible evitar el accidente, incluso después de la caída de la primera pieza del dominó, si fuese retirada una de las piezas de la secuencia, o sea, los actos inseguros. 
Figura 1 - Teoría de la causa única.

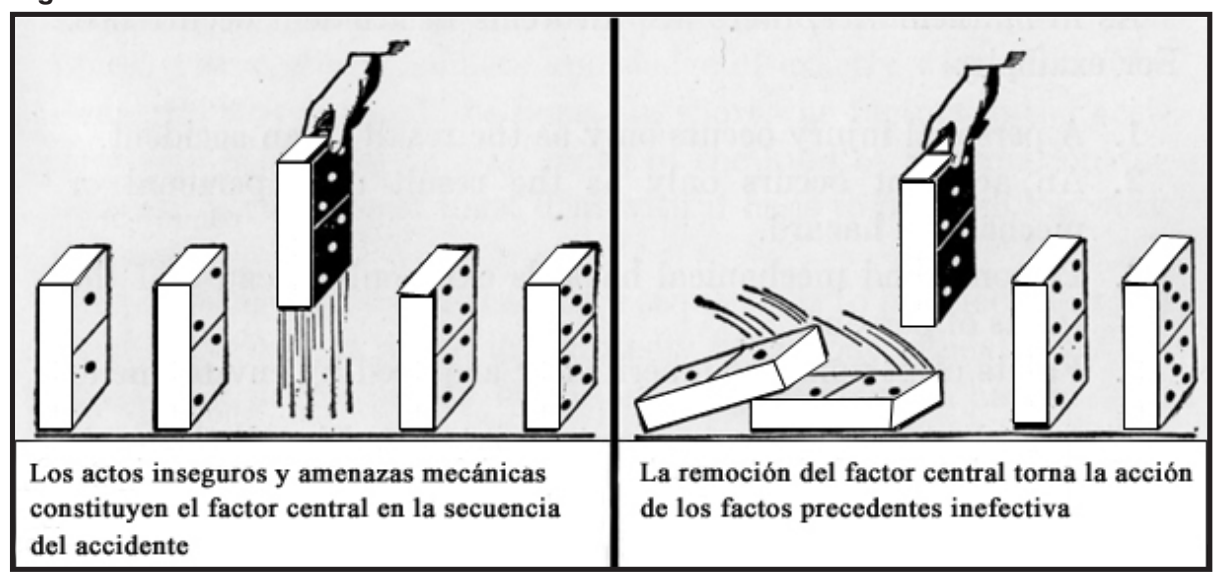

Fuente: Adaptado de Heinrich (1931).

El modelo de Reason (1997), conocido como "Queso Suizo" o teoría de las causas múltiples, no defiende una causa única como desencadenadora de una secuencia de eventos que llevaría al accidente, sino combinaciones lineales de condiciones latentes y fallas activas que constituyen varias cadenas y, después de superar las barreras de seguridad por la alineación de sus vulnerabilidades, culminan en el accidente, conforme Figura 2. Reason (1997) destaca la influencia de la organización en la ocurrencia de los accidentes. Así, las investigaciones deben procurar condiciones latentes que puedan inducir a situaciones propicias para las fallas activas. De esa forma, la prevención más efectiva debería identificar peligros o amenazas y administrar los riesgos (REASON, 1997).

Reason (1997), a pesar de haberse preocupado en investigar aspectos organizacionales y la influencia de los más elevados niveles administrativos como factores contribuyentes, mantuvo la falla activa defendida por Heinrich (1931) en la descripción de la ocurrencia del accidente. De esa forma, no hubo una total ruptura, sino una evolución de paradigma que, de cierta forma, se apoya en el anterior.

Actualmente, hay una tendencia de representar el accidente por medio de un modelo sistémico. Según Hollnagel (2004), la adaptación del modelo de Rasmussen (1997) originó esta nueva perspectiva, en la cual el accidente no sería más analizado bajo una óptica de linealidad, sino por múltiples factores de actuación desordenada y simultánea.

Hollnagel (2004) defiende que un sistema varía conforme un modelo estocástico, pues la manifestación de esas variaciones es aleatoria e indeterminada, pero la sumatoria de las variaciones, actuando simultáneamente, puede potencializar la probabilidad de los accidentes.

Figura 2 - Teoría de las causas múltiples.

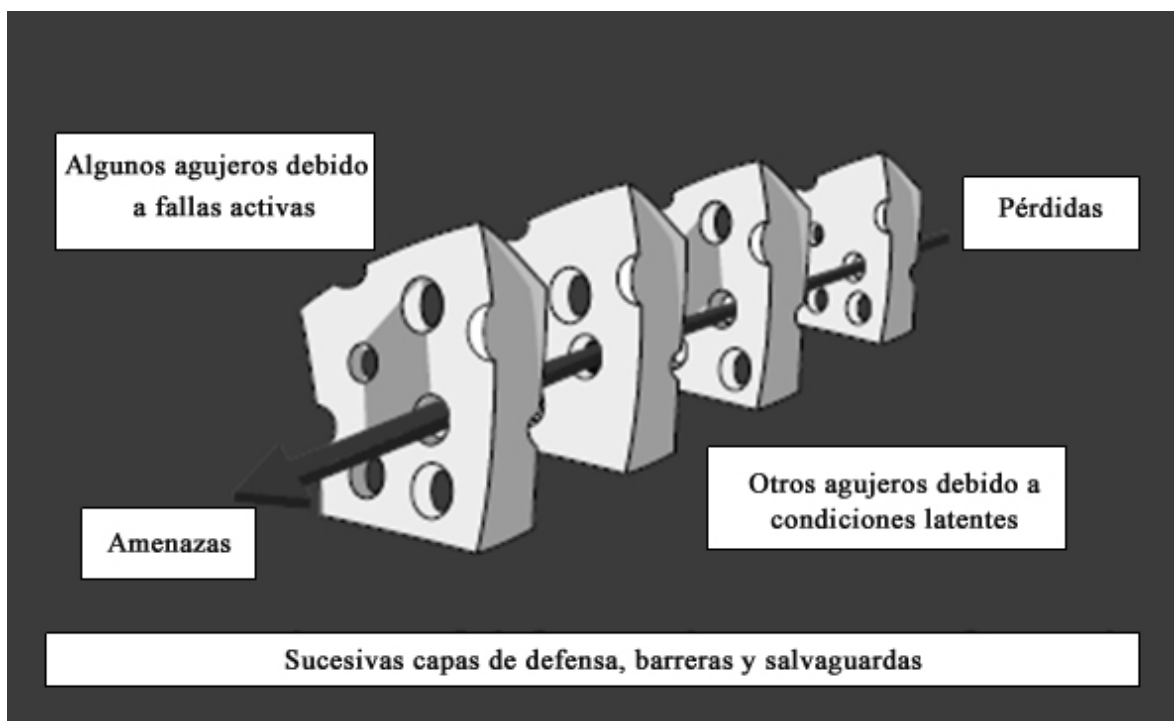

Fuente: Adaptado de Reason (1997). 
En la visión de Hollnagel (2004), las investigaciones de accidentes no deben procurar una relación de causaefecto para crear defensas o barreras, pues las variaciones en las interacciones internas tornan ineficaz ese abordaje para prevención de accidentes. De esa forma, sería más adecuado investigar todo el sistema, sus interacciones y variaciones, para que la prevención sea basada en el acompañamiento de la variabilidad y en la capacidad de adaptación a las presiones, considerando factores que pueden actuar en el sistema de manera estocástica y resonante. La resiliencia se torna el principal criterio de seguridad, en virtud de la necesidad de adaptación a los cambios, de la flexibilidad de los sistemas complejos y de la imprescindible capacidad de retornar al equilibrio después de una inestabilidad.

El modelo Sistémico, aunque no sea lineal, no representa una total ruptura con las representaciones anteriores, pues los diversos factores que ejercen influencia en la seguridad continúan relevantes durante las investigaciones.

\section{CONCLUSIÓN}

Las investigaciones de accidentes aeronáuticos son conducidas conforme las teorías que buscan explicar esas ocurrencias. De esa forma, la evolución de paradigmas sobre los asuntos relacionados a la seguridad de vuelo, como error humano, factores contribuyentes y causas, provoca también la evolución en los métodos investigativos.

En la historia de la seguridad de vuelo, cuando un conjunto teórico no explica más satisfactoriamente los accidentes y no reduce las estadísticas, nuevas teorías surgen y proponen una mejor adecuación a la realidad.

Siendo así, la evolución de la investigación de accidentes aeronáuticos puede ser explicada a la luz de la teoría elaborada por Kuhn (1991), ya que las ciencias aeronáuticas también sufren evoluciones paradigmáticas, sin ruptura total en la estructura conceptual.

\section{REFERENCIAS}

BRASIL. Comando da Aeronáutica. Gabinete do Comando da Aeronáutica. Portaria no 2.230/GC3, de 23 de dezembro de 2013. Aprova a reedição da NSCA 3-6, que dispõe sobre a Investigação de Ocorrências Aeronáuticas com Aeronaves Militares (NSCA 3-6). Boletim do Comando da Aeronáutica, Brasília, DF, n. 248, f. 12148, 30 dez. 2013.

Comando da Aeronáutica. Gabinete do Comando da Aeronáutica. Portaria nำ166/GC3, de 12 de fevereiro de 2014. Aprova a reedição da NSCA 3-13, que dispõe sobre os Protocolos de Investigação de Ocorrências Aeronáuticas da Aviação Civil conduzida pelo Estado Brasileiro (NSCA 3-13) Boletim do Comando da Aeronáutica, Brasília, DF, n. 034, f. 1285, 18 fev. 2014.

Decreto $n^{\circ}$ 24.749, de 05 de abril de 1948 . Aprova o Regulamento para o Serviço de Investigação de Acidentes Aeronáuticos. Diário Oficial da União. Brasília, DF, 07 abr. 1948. Seção 1, p. 5523.

Decreto $\mathrm{n}^{\circ}$ 57055, de 11 de novembro de 1965. Aprova o Regulamento para o Serviço de Investigação e Prevenção de Acidentes Aeronáuticos. Diário Oficial da União. Brasília, DF, 19 out. 1965. Seção 1, p. 10636.

Lei $\mathrm{n}^{\circ}$ 12.970, de 8 de maio de 2014. Dispõe sobre as investigações do Sistema de Investigação e Prevenção de Acidentes

Aeronáuticos - SIPAER e o acesso aos destroços de aeronave. Diário Oficial da União. Brasília, DF, 09 maio 2014. p. 1.

EBER, D. H. F. F. Sciences, v. 22, n. 4, p. 15-19, 1982.

HEINRICH, H. W. Industrial accident

prevention: a scientific approach. New York: McGraw-Hill, 1931.

HOLLNAGEL, E. Barrier analysis and accident prevention. Aldershot, UK: Ashgatee, 2004.

INTERNATIONAL CIVIL AVIATION ORGANIZATION. Doc 9859: Safety Management Manual (SMM). 3. ed. Montreal, 2013.

INTERNATIONAL CIVIL AVIATION ORGANIZATION. Annex 19: Safety Management. Montreal, 2013.

KUHN, T. S. A estrutura das revoluções científicas. São Paulo: Perspectiva, 1991.

REASON, J. Managing the risks of organizational accidents. Burlington: Ashgate, 1997.

STOLZER, A. J.; HALFORD, C. D.; GOGLIA, J. J. Safety management systems in aviation. Burlington: Ashgate, 2008. 